\title{
Tinnitus, Hörsturz und Vertigo
}

B ei Tinnitus und Schwindel handelt es sich nicht um einheitliche Krankheitsbilder, sondern um unspezifische Symptome, die bei einer Reihe verschiedener Erkrankungen auftreten. Im akuten Stadium stellen sie Alarmsignale dar, die einer umfassenden interdisziplinären Abklärung bedürfen.

Fast jeder Mensch kennt beide Symptome: Ein hochfrequentes Klingen in den Ohren nach einer Disko-Nacht, Bohren in der Betonwand, um ein Bild aufzuhängen, oder Arbeiten mit der Kreissäge, aber auch nach einem Hörsturz oder Menière-Anfall bzw. Knalltrauma ist ein weitverbreitetes Phänomen. Da es bisher keine epidemiologischen Daten für Deutschland gab, hat die Selbsthilfeorganisation Deutsche Tinnitus-Liga (DTL, Wuppertal) 1998 aus überwiegend eigenen Mitteln ihrer 20.000 tinnitusbetroffenen Mitgliedern eine repräsentative Studie initiiert. Danach kommt es jährlich bei zehn Millionen Mitbürgern zu akutem Tinnitus, der bei ca. 250000 chronifiziert. Altersabhängig kommt es zu einer Zunahme der Prävalenz mit einem Gipfel bis $11 \%$ bei den 60 - bis 80 -jährigen. Auch schwindelerregende Situationen sei es beim Bootfahren oder Bergsteigen oder im Rahmen einer Erkrankung sind jedem bekannt. Auch hier findet sich eine mit dem Alter zunehmende Prävalenz von 19 bis 39\%.

Die in diesem Heft im Fokus stehenden Symptome sind teilweise verschwistert, teilweise nur Nachbarn. Störungen im endolymphatischen System des Innenohrs und dessen Vernetzungen mit dem Vestibularapparat verursachen die von Prosper Menière bereits 1861 beschriebene intermittierende Symptomtrias Tinnitus, Hörsturz und Schwindel. Auch der gemeinsam verlaufende Verbindungsabschnitt als 8. Hirnnerv sind Gründe, warum das Akustikusneurinom (Vestibularisschwannom) oder Felsenbeinfrakturen zu Läsionen führen, die Innenohr und Gleichgewichtsorgan gleichermaßen betreffen. Andererseits kommen unspezifische Schwindelformen und Tinnitus gemeinsam, aber völlig unabhängig voneinander vor. Gerade dies macht ein differenziertes Vorgehen erforderlich, an dem HNOArzt und Neurologe in unterschiedlicher Gewichtung beteiligt sind.

Unabhängig von den Ursachen und dem Alter können Tinnitus und Schwindel die Lebensqualität in erheblichem Maß beeinträchtigen und zu einer allgemeinen Verunsicherung beitragen. Der 1981 in einer HNO-Zeitschrift gemachte Hinweis von Jobst Böning, Würzburg, „dass der HNO-Arzt der Psyche näher sitzt, als der Psychiater“", karikiert die Komplexheit, mit der sich die zwei Fachdisziplinen aber auch Allgemeinärzte, Internisten, Orthopäden und Zahnärzte mit Tinnitus und Schwindel konfrontiert sehen. Dass Tinnitus und Schwindel auch Symptome psychischer Erkrankungen sein können, wird viel zu wenig berücksichtigt: So findet sich z.B. bei Patienten mit Panikstörung bei bis zu 56\% Tinnitus und 44\% Schwindel. Sogar 78\% der Patienten mit Angststörungen geben Schwindel als eines ihrer vordergründigen Symptome an. Auch bei somatoformen Störungen (vor allem bei Somatisierungsstörungen und Hypochondrie) finden sich auffallend häufig Patienten, die Tinnitus (bis $42 \%$ ) und Schwindel als eines unter vielen sie stark beunruhigenden Beschwerden angeben. Dies geht so weit, dass in einer neurootologischen Spezialambulanz bei knapp zwei Drit- tel der dort untersuchten Patienten sich gegenwärtig oder zurückliegend psychische Störungen finden lassen, wenn sie erfragt werden.

Nicht selten entwickeln sich in Folge einer primären vestibulären Erkrankung (z.B. M. Menière, benigner paroxysmaler Lagerungsschwindel) oder eindeutigem otogenen Tinnitus (z.B. nach Hörsturz, Knalltrauma, etc.) bei differenzierter Untersuchung Angst- oder Depressionserkrankungen, die entscheidend bei der Aufrechterhaltung der Symptomatik beteiligt sind bzw. die Therapieresistenz der somatisch ausgerichteten Behandlung erklären. Entsprechend frustrierend ist der Verlauf bei diesen Patienten, die oft erst nach Jahren nach einer fachübergreifenden psychiatrisch/psychotherapeutischen Diagnostik den Weg zu einer entsprechenden Behandlung finden. Die dann eingeleitete Therapie der Angst-, Depressions- und Somatoformen Störung vermag einen drastischen Rückgang der Tinnitusqual und Schwindelsymptomatik bewirken.

Das vorliegende Heft soll daher einen Überblick über spezielle Entwicklungen bei Tinnitus, Hörsturz und Schwindel (siehe auch psychoneuro 12/2003) geben. Die Beiträge betonen die Notwendigkeit, dass vor allem bei der Chronifizierung die Vernetzung der psychosomatischen Versorgung mit den beteiligten Fachdisziplinen einen großen Nachholbedarf hat. Nach wie vor begegnen wir allzu vielen Betroffenen mit einer oft jahrelangen Odyssee auf der Suche nach dem Verstehen ihrer Symptomatik. So möchte diese Themenzusammenstellung ein vertieftes Verständnis dieser alltäglichen „unsichtbaren Leiden“ fördern.

Es ist zu wünschen, dass dieser unselige Verabschiedungssatz „Da kann man nichts machen“ die Betroffenen immer seltener zu hören bekommen und anstelle der zu häufigen AlibiRezeptur von „Durchblutungstabletten“ mehr Ermutigung und Anleitung zur Selbsthilfe (Deutsche Tinnitus-Liga, www. tinnitus-liga.de) erfolgt und die Hinweise an die Hand bekommen, wie sie ihre Lebensqualität weitgehend wiederherstellen können.

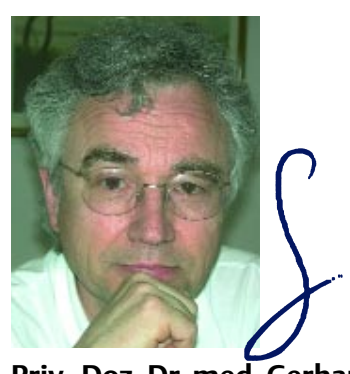

Priv.-Doz. Dr. med. Gerhard Goebel, Roseneck 